\title{
Thermal networks from the heat equation by using the finite element method
}

\author{
I. Naveros ${ }^{1,2,3}$, C. Ghiaus ${ }^{3}$, J. Ordoñez ${ }^{2}$ \& D. P. Ruíz ${ }^{1}$ \\ ${ }^{1}$ Department of Applied Physics, University of Granada, Spain \\ ${ }^{2}$ CETHIL UMR5008, INSA-Lyon, France \\ ${ }^{3}$ Department of Civil Engineering, University of Granada, Spain
}

\begin{abstract}
The classical heat equation is a partial differential equation (PDE) which is utilized for describing heat conduction in solids considering continuous media; the heat equation is stated taking into account the principle of energy conservation and Fourier's law. Thermal networks are usually used for studying heat transfer processes by considering a thermal-electrical analogy, as it is noted by Carslaw and Jaeger, despite the fact that well-known discrepancies between heat and electricity exist. On the other hand, numerical prediction of thermal problems using finite elements has been developed significantly and numerical codes like ANSYS fluent or COMSOL are widely used to solve heat conduction in combination with fluid mechanical problems. The aim of this paper is to show that thermal networks may be deduced from the heat equation without the thermalelectrical analogy by using finite elements. For this purpose, the heat equation is put in its weak form and, by using the finite element method as introduced by Gilbert Strang, the weak form of the heat equation is expressed as a system of differential and algebraic equations (DAE), i.e. as a thermal network.
\end{abstract}

Keywords: heat equation, thermal networks, finite elements, model transformations.

\section{Introduction}

The classical heat equation, which is a deterministic parabolic partial differential equation (PDE), is usually employed for modelling heat conduction in solids. It was introduced by Fourier in his celebrated "Théorie Analytique de la Chaleur" for studying heat diffusion from a continuous standpoint $[1,2]$. Furthermore, 
Fourier developed analytical methods for solving the heat equation which are still employed [3, 4].

On another hand, numerical methods became years later and they are mainly used for prediction of thermal problems, among them, finite elements have been developed significantly and numerical codes like ANSYS $^{\circledR}$ fluent or COMSOL ${ }^{\circledR}$ are widely used to solve heat conduction in combination with fluid mechanical problems. The use of these software tools in computational fluid dynamics (CFD) is mainly focused on simulation problems, and parameter identification problems are not considered. Nonetheless, parameter identification is an important issue which may be also dealt with finite element techniques and this can be noted explicitly if thermal networks are deduced from the heat equation.

The deduction of thermal networks from the heat equation supposes to follow the inverse way that Fourier followed, i.e. to discretize the heat equation for solving it. Thermal networks usually appear considering the analogy with electrical networks [5-7], as noted by Carslaw and Jaeger [3]. Thus, it is important to highlight that the analogy between heat conduction and electricity started in the inverse way, i.e. Fourier's law preceded Ohm's law as noted within an historical review by Narasimhan [2]. This paper shows the deduction of thermal networks from the heat equation by using the finite element method $[8,9]$.

\section{Transforming the heat equation into a thermal network}

The heat equation can be expressed as a system of linear differential and algebraic equations (DAE) using the Galerkin finite element method, as shown for systems in equilibrium by Strang $[8,9]$, and a system of DAE can be represented as a thermal network (Figure 1), as shown by Ghiaus [10], Naveros and Ghiaus [11] and Naveros et al. [12].

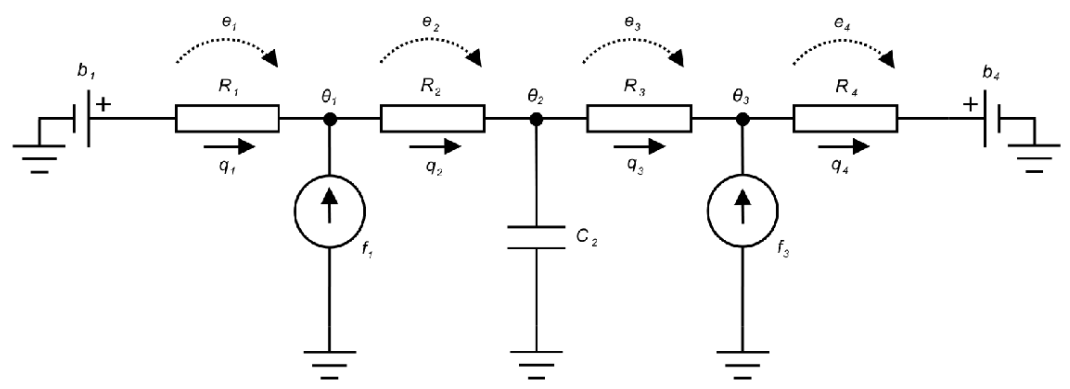

Figure 1: A thermal network which represent a system of DAE with one differential and two algebraic equations.

In Figure $1, b_{1}$ and $b_{4}$ are temperature sources which act as boundary conditions, $f_{1}$ and $f_{3}$ are heat rate sources which act as boundary conditions, $\theta_{1}, \theta_{2}$, and $\theta_{3}$ are temperatures at nodes, $R_{1}, R_{2}, R_{3}$ and $R_{4}$ are thermal resistances 
in branches from node to node, $q_{1}, q_{2}, q_{3}$ and $q_{4}$ are heat rate fluxes over branches, and $C_{2}$ is the thermal capacity at node $\theta_{2}$.

Let the heat equation for a continuous isotropic non-homogeneous medium:

$$
\rho c \frac{\partial \theta}{\partial t}=-\nabla \cdot(-\kappa \nabla \theta)+p
$$

where $\rho=\rho(x, y, z)$ is the volume density, $c=c(x, y, z)$ is the volume specific heat capacity, $\kappa=\kappa(x, y, z)$ is the thermal conductivity of the volume, $p=$ $p(x, y, z)$ is a function which includes heat rate sources supplied to the volume, and $\theta=\theta(x, y, z)$ is the function of temperature distribution in the volume.

The heat equation, Eq. (1), may be expressed in weak form and the finite element method may be used for solving it. In three-dimensions, the weak form of the heat equation is:

$$
\int_{V} \rho c \frac{\partial \theta}{\partial t} v \mathrm{~d} V=\int_{V}-\nabla \cdot(-\kappa \nabla \theta) v \mathrm{~d} V+\int_{V} p v \mathrm{~d} V
$$

where the heat equation is integrated over the volume, $V$, using a test function $v=$ $v(x, y, z)$.

Firstly, the divergence and gradient operators may be developed considering Cartesian coordinates:

$$
\begin{aligned}
\int_{V} \rho c \frac{\partial \theta}{\partial t} v \mathrm{~d} V=\int_{V} & \left(\frac{\partial}{\partial x}\left(\kappa_{x} \frac{\partial \theta}{\partial \mathrm{x}}\right)+\frac{\partial}{\partial y}\left(\kappa_{y} \frac{\partial \theta}{\partial \mathrm{y}}\right)+\frac{\partial}{\partial z}\left(\kappa_{z} \frac{\partial \theta}{\partial \mathrm{z}}\right)\right) v \mathrm{~d} V \\
+ & \int_{V} p v \mathrm{~d} V
\end{aligned}
$$

This integral expression becomes a system of differential algebraic equations (DAE), and represented as a thermal network graphically. Next, the part corresponding to the $x$-coordinate is used for detailing how obtaining the matrix form, the extension to three dimensions may be done considering a test function $v=v(x)+v(y)+v(z)$.

For one dimension considering the total interval $x \in[0,1]$, we obtain:

$$
\begin{aligned}
\int_{S} \operatorname{dydz} \int_{0}^{1} \rho c \frac{\partial \theta}{\partial t} & v \mathrm{dx} \\
& =\int_{S} \mathrm{dydz} \int_{0}^{1} \frac{\partial}{\partial x}\left(\kappa_{x} \frac{\partial \theta}{\partial \mathrm{x}}\right) v \mathrm{dx}+\int_{S} \mathrm{dydz} \int_{0}^{1} p v \mathrm{dx}
\end{aligned}
$$

Moreover, by considering the integration into a cube of volume: $\mathrm{V}=\int_{V} \mathrm{dxdydz}=h^{3}$, the surface perpendicular to the $x$-coordinate will be, $S=$ $\int_{S} \mathrm{dydz}=h^{2}$, and Eq. (4) becomes:

$$
h^{2} \int_{0}^{1} \rho c \frac{\partial \theta}{\partial t} v \mathrm{dx}=h^{2} \int_{0}^{1} \frac{\partial}{\partial x}\left(\kappa_{x} \frac{\partial \theta}{\partial \mathrm{x}}\right) v \mathrm{dx}+h^{2} \int_{0}^{1} p v \mathrm{dx}
$$


Following, Eq. (5) will be put in matrix notation by using the finite element Galerkin method as explained by Strang [9]. For this purpose, a continuous piecewise linear approximation for building the trial functions $\varphi_{i}(x)$ is used (Figure 3). Let $I_{h}: 0=x_{1}<x_{2}<\cdots<x_{N}=1$ be a partition of interval $I=(0,1)$ into subintervals $I_{j}=\left(x_{j}, x_{j-1}\right)$ of length $h_{j}=x_{j}-x_{j-1}$; we will suppose for the sake of simplicity $h_{j}=h$. Let $V_{h}$ denote the set of continuous piecewise linear functions on $I_{h}$ that are one at $x=0$ and $x=1 . V_{h}$ is a finite dimensional vector space, $\operatorname{dim} V_{h}=N$, with a basis consisting of hat functions $\left\{\varphi_{i}\right\}_{i=1}^{N}$ :

$$
\varphi_{i}(x)=\left\{\begin{array}{cl}
0, & \text { if } x \notin\left[x_{i-1}, x_{i+1}\right], \\
\frac{x-x_{i-1}}{x_{i}-x_{i-1}}, & \text { if } x \in\left[x_{i-1}, x_{i}\right], \\
\frac{x_{i+1}-x}{x_{i+1}-x_{i}}, & \text { if } x \in\left[x_{i}, x_{i+1}\right]
\end{array}\right.
$$

Using such trial functions, the temperature function may be defined as:

$$
\theta=\sum_{i=1}^{N} \theta_{i} \varphi_{i}(x)
$$

and the test function may be defined as a set of $N$ functions at each space interval by:

$$
v_{j}=\varphi_{j}(x) j=2, \ldots, N-1 ; v_{1}=0 ; v_{N}=0
$$

These test functions will coincide with hat functions except at interval extremes $\left(v_{1} \neq \varphi_{1}, v_{N} \neq \varphi_{N}\right)$.

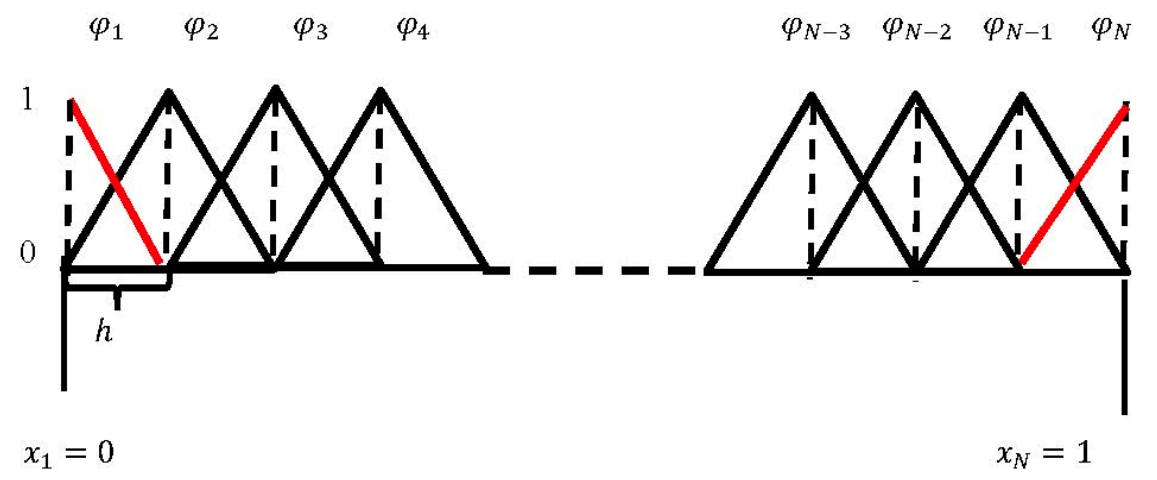

Figure 2: Trial hat functions and semi-hat functions at extremes.

The function of thermal conductivities may be also defined by parts:

$$
\left.\kappa(x)=\kappa_{i} \quad \text { for } x \in\right] x_{i-1}, x_{i}[, \quad i=1, \ldots, N-1
$$

It needs to be noted that thermal conductivity does not need to be a continuous function but the heat flux and the temperature distribution. At interval junctions, the thermal conductivity may change without continuity since the space may be non-homogenous, for instance when there is a change of material [3, 9]. 
Next, we consider the integration by parts of the first term in the right-hand side of Eq. (5):

$$
h^{2} \int_{0}^{1} \frac{\mathrm{d}}{\mathrm{dx}}\left(\kappa_{x} \frac{\mathrm{d} \theta}{d x}\right) v \mathrm{~d} x=-h^{2} \int_{0}^{1} \kappa_{x} \frac{\mathrm{d} \theta}{\mathrm{dx}} \frac{\mathrm{d} v}{d x} \mathrm{~d} x+\left.h^{2} \kappa \frac{\mathrm{d} \theta}{d x} v\right|_{0} ^{1}
$$

The second term in the right-hand side becomes zero by choosing the test function $v(0, y, z)=0$ and $v(1, y, z)=0$; the first term in the right-hand side of Eq. (10) may be written as $N$ expressions $(j=1, \ldots, N)$ and each expression can be expanded in $N$ terms $(i=1, \ldots, N)$ considering the previously proposed test and the trial functions $\left(v_{j}, \varphi_{i}\right)$ :

$$
\left.\begin{array}{c}
N \text { trial functions }\left(\varphi_{i}\right) \\
-h^{2}\left(\frac{\mathrm{d} \varphi_{1}}{\mathrm{~d} x} \theta_{1}+\cdots+\frac{\mathrm{d} \varphi_{N}}{\mathrm{~d} x} \theta_{N}\right)\left(\frac{\mathrm{d} v_{1}}{\mathrm{~d} x}\right) \int_{0}^{1} \kappa d x \\
\vdots \\
-h^{2}\left(\frac{\mathrm{d} \varphi_{1}}{\mathrm{~d} x} \theta_{1}+\cdots+\frac{\mathrm{d} \varphi_{N}}{\mathrm{~d} x} \theta_{N}\right)\left(\frac{\mathrm{d} v_{N}}{\mathrm{~d} x}\right) \int_{0}^{1} \kappa d x
\end{array}\right\} N \text { test functions }\left(v_{j}\right)
$$

This enables us to use matrix notation for rewriting expression (11) as $\mathbf{K} \boldsymbol{\theta}_{\varphi}$, where $\boldsymbol{\theta}_{\varphi}=\left(\theta_{1} \ldots \theta_{n}\right)^{T}$ is the vector of temperatures representing state variables and temperature sources; and $\mathbf{K}$ is the stiffness matrix, which is a sparse tridiagonal matrix $(N \times N)$ with elements different to zero only on the diagonal and on the adjacent diagonals. Furthermore, this stiffness matrix will be symmetric and positive definite hence invertible.

In practice, for obtaining the stiffness matrix the test and trial functions, which are piece-wise functions, may be arranged as vectors and matrices. The total interval $[0,1]$ may be split into $N-1$ subintervals, which may be supposed equal spaced with a length $h$, and each subinterval will represent a vector component. For instance, the derivatives of a set of trial functions, Eq. (6), considering $N=5$, may be represented as five independent column vectors and arranged as a matrix (Figures 3-7 and Eqs (12)-(18)).

The derivative of the first trial function $\left(\varphi_{1}^{\prime}\right)$ (Figure 3), may be defined for each interval as:

$$
\begin{aligned}
& \boldsymbol{\varphi}_{1}^{\prime}=\frac{1}{h}\left(\begin{array}{llll}
-1 & 0 & 0 & 0
\end{array}\right)^{T} \\
& \varphi_{1}
\end{aligned}
$$

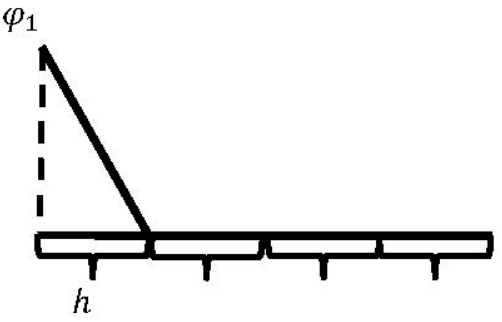

Figure 3: First trial function (semi-hat function) using four intervals of length $h$. 
The derivative of the second trial function $\left(\varphi_{2}^{\prime}\right)$ considered in Figure 4 may be defined for each interval as $1 / \mathrm{h},-1 / \mathrm{h}, 0$ and 0 respectively:

$$
\boldsymbol{\varphi}_{2}^{\prime}=\frac{1}{h}\left(\begin{array}{llll}
1 & -1 & 0 & 0
\end{array}\right)^{T}
$$

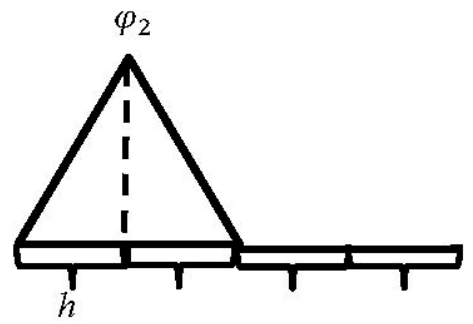

Figure 4: Second trial function (hat function) using four intervals of length $\mathrm{h}$.

The derivative of the third trial function $\left(\varphi_{3}^{\prime}\right)$ considered in Figure 5 may be defined for each interval as $0,1 / \mathrm{h},-1 / \mathrm{h}$ and 0 respectively:

$$
\boldsymbol{\varphi}_{3}^{\prime}=\frac{1}{h}\left(\begin{array}{llll}
0 & 1 & -1 & 0
\end{array}\right)^{T}
$$

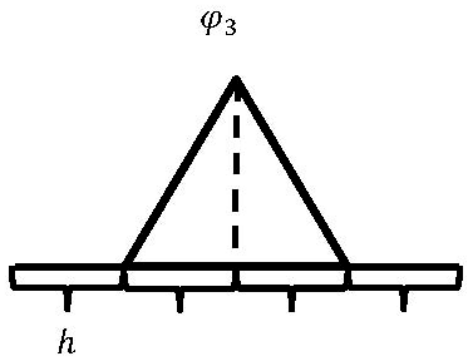

Figure 5: Third trial function (hat function) using four intervals of length $h$.

The derivative of the fourth trial function $\left(\varphi_{4}^{\prime}\right)$ considered in Figure 6 may be defined for each interval as $0,0,1 / \mathrm{h}$ and $-1 / \mathrm{h}$ respectively:

$$
\boldsymbol{\varphi}_{4}^{\prime}=\frac{1}{h}\left(\begin{array}{llll}
0 & 0 & 1 & -1
\end{array}\right)^{T}
$$




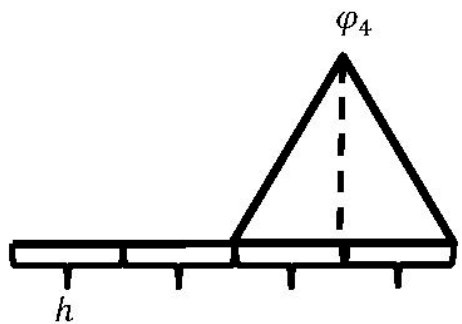

Figure 6: Fourth trial function (hat function) using four intervals of length $h$.

The derivative of the fifth trial function $\left(\varphi_{5}^{\prime}\right)$ considered in Figure 7 may be defined for each interval as $0,0,0$ and $1 / \mathrm{h}$ respectively:

$$
\boldsymbol{\varphi}_{5}^{\prime}=\frac{1}{h}\left(\begin{array}{llll}
0 & 0 & 0 & 1
\end{array}\right)^{T}
$$

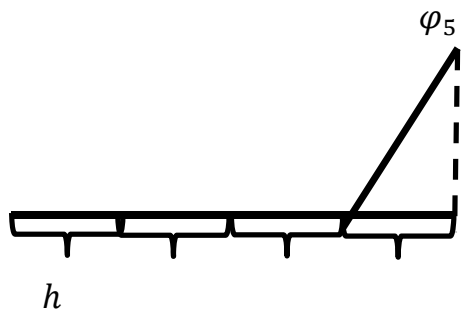

Figure 7: Fifth trial function (semi-hat function) using four intervals of length $h$.

The trial functions may be arranged altogether as column vectors to form a difference matrix, $\mathbf{A}_{\varphi}$ :

$$
\mathbf{A}_{\varphi}=\frac{1}{h}\left(\begin{array}{rrrrr}
\boldsymbol{\varphi}_{1}^{\prime} & \boldsymbol{\varphi}_{2}^{\prime} & \boldsymbol{\varphi}_{3}^{\prime} & \boldsymbol{\varphi}_{4}^{\prime} & \boldsymbol{\varphi}_{5}^{\prime} \\
\hline-1 & 1 & 0 & 0 & 0 \\
0 & -1 & 1 & 0 & 0 \\
0 & 0 & -1 & 1 & 0 \\
0 & 0 & 0 & -1 & 1
\end{array}\right)
$$

On another hand, the test functions are equals to the trial functions except at interval extremes where they may be imposed to be zero for cancelling the second term on the right-hand side of Eq. (10). This second term is related to the boundary conditions used for solving the problem and it needs to be cancelled for obtaining an invertible stiffness matrix, which is similar to the use of boundary conditions after an analytical integration for obtaining the integration constants. The difference matrix, $\mathbf{A}_{v}$, obtained from test functions is: 


$$
\mathbf{A}_{v}=\frac{1}{h}\left(\begin{array}{rrrrr}
v_{1}^{\prime} & v_{2}^{\prime} & v_{3}^{\prime} & v_{4}^{\prime} & v_{5}^{\prime} \\
\hline 0 & 1 & 0 & 0 & 0 \\
0 & -1 & 1 & 0 & 0 \\
0 & 0 & -1 & 1 & 0 \\
0 & 0 & 0 & -1 & 0
\end{array}\right)
$$

Thermal conductivities may be arranged in a diagonal matrix, $\boldsymbol{\Lambda}$, considering they can be different for each interval $(N-1=4)$ of length $h$.

$$
\boldsymbol{\Lambda}=\left(\begin{array}{rrrr}
\kappa_{1} & 0 & 0 & 0 \\
0 & \kappa_{2} & 0 & 0 \\
0 & 0 & \kappa_{3} & 0 \\
0 & 0 & 0 & \kappa_{4}
\end{array}\right)
$$

In this way, expression (11) may be written using matrix notation as:

$$
-h^{2} \mathbf{A}_{v}^{T} h \mathbf{\Lambda} \mathbf{A}_{\varphi} \boldsymbol{\theta}_{\varphi}
$$

where the term $h \boldsymbol{\Lambda}$ comes from the integral given in expression (11), which is integrated interval by interval:

$$
\int_{0}^{1} \kappa \mathrm{dx}=\int_{0}^{h} \kappa_{1} \mathrm{dx}+\int_{h}^{2 h} \kappa_{2} \mathrm{dx}+\int_{2 h}^{3 h} \kappa_{3} \mathrm{dx}+\int_{3 h}^{1} \kappa_{4} \mathrm{dx}
$$

With this configuration $(N=5)$, we have five trial functions, $\varphi_{i}$, and five test functions, $v_{j}$, but only three test functions $\left(v_{2}, v_{3}, v_{4}\right)$ are different from zero. Hence three equations obtained from expression (20) are only different from zero:

$$
-\left(\begin{array}{rrrr}
0 & 0 & 0 & 0 \\
1 & -1 & 0 & 0 \\
0 & 1 & -1 & 0 \\
0 & 0 & 1 & -1 \\
0 & 0 & 0 & 0
\end{array}\right) h\left(\begin{array}{rrrr}
\kappa_{1} & 0 & 0 & 0 \\
0 & \kappa_{2} & 0 & 0 \\
0 & 0 & \kappa_{3} & 0 \\
0 & 0 & 0 & \kappa_{4}
\end{array}\right)\left(\begin{array}{ccccc}
-1 & 1 & 0 & 0 & 0 \\
0 & -1 & 1 & 0 & 0 \\
0 & 0 & -1 & 1 & 0 \\
0 & 0 & 0 & -1 & 1
\end{array}\right)\left(\begin{array}{c}
\theta_{\varphi 1} \\
\theta_{\varphi 2} \\
\theta_{\varphi 3} \\
\theta_{\varphi 4} \\
\theta_{\varphi 5}
\end{array}\right)=h\left(\begin{array}{c}
0 \\
\kappa_{1}\left(\theta_{\varphi 1}-\theta_{\varphi 2}\right)+\kappa_{2}\left(\theta_{\varphi 3}-\theta_{\varphi 2}\right) \\
\kappa_{2}\left(\theta_{\varphi 2}-\theta_{\varphi 3}\right)+\kappa_{3}\left(\theta_{\varphi 4}-\theta_{\varphi 3}\right) \\
\kappa_{3}\left(\theta_{\varphi 3}-\theta_{\varphi 4}\right)-\kappa_{4}\left(\theta_{\varphi 5}-\theta_{\varphi 4}\right) \\
0
\end{array}\right)
$$

The result obtained in Eq. (22) may be rearranged as:

$$
-\mathbf{A}^{T} \mathbf{G A \theta}+\mathbf{A}^{\mathbf{T}} \mathbf{G b}
$$

where the vector of temperatures, $\boldsymbol{\theta}_{\varphi}=\left(\theta_{\varphi 1} \theta_{\varphi 2} \theta_{\varphi 3} \theta_{\varphi 4} \theta_{\varphi 5}\right)^{T}$, is split in a vector of temperatures representing the state-variables, $\boldsymbol{\theta}=\left(\theta_{1} \theta_{2} \theta_{3}\right)^{T}$, and in a vector of temperature sources (boundary conditions), $\mathbf{b}=\left(\begin{array}{llll}b_{1} & 0 & 0 & b_{4}\end{array}\right)^{T}$. For this, the next change of variables is considered: $\theta_{\varphi 1} \equiv b_{1}, \theta_{\varphi 2} \equiv \theta_{1}, \theta_{\varphi 3} \equiv \theta_{2}$ and $\theta_{\varphi 4} \equiv \theta_{3}$ and $\theta_{\varphi 5} \equiv-b_{4}$. A is a difference matrix where all the columns are independent, which guarantees that the stiffness matrix $\left(\mathbf{K} \equiv-\mathbf{A}^{T} \mathbf{G A}\right)$ is invertible: 


$$
\mathbf{A}=\left(\begin{array}{rrr}
1 & 0 & 0 \\
-1 & 1 & 0 \\
0 & -1 & 1 \\
0 & 0 & -1
\end{array}\right)
$$

and $\mathbf{G}$ is a matrix of thermal conductances, which is obtained by multiplying the length of each interval, $h$, for the matrix of thermal conductivities, $\Lambda$ :

$$
\mathbf{G}=h \boldsymbol{\Lambda}=\left(\begin{array}{rrrr}
R_{1}^{-1} & 0 & 0 & 0 \\
0 & R_{2}^{-1} & 0 & 0 \\
0 & 0 & R_{3}^{-1} & 0 \\
0 & 0 & 0 & R_{4}^{-1}
\end{array}\right)
$$

Next, the terms which refer the heat rate sources and the energy accumulation in Eq. (5) may be put altogether as:

$$
h^{2} \int_{0}^{1}\left(\rho c \frac{\partial \theta}{\partial t}-p\right) v \mathrm{~d} x
$$

The expression (26) may also be written in matrix notation. For instance, by making the integration using the Lagrange interpolation method:

$$
\int_{0}^{1} g(x) v_{i}(x) \mathrm{d} x \approx g\left(x_{i}\right) \int_{0}^{1} v_{i}(x) \mathrm{d} x
$$

where in our case for $g=h^{2}(\rho c \dot{\theta}-p)$.

Eq. (27) may be split in five terms corresponding to five test functions $(N=$ 5 ), but only three test functions will be different from zero as in Eq. (22). This allows to define the matrix of thermal capacities, $\mathbf{C}$, as:

$$
\mathbf{C}=\left(\begin{array}{ccc}
C_{1} & 0 & 0 \\
0 & C_{2} & 0 \\
0 & 0 & C_{3}
\end{array}\right)
$$

where $C_{i}=\rho_{i} c_{i} h^{3}$; and the vector of heat rate sources is defined as:

$$
\mathbf{f}=\left(f_{1} f_{2} f_{3}\right)^{T}
$$

where $f=p_{i} h^{3}$.

The resulting matrix expression is:

$$
\mathbf{C} \dot{\boldsymbol{\theta}}-\mathbf{f}
$$

where $\dot{\boldsymbol{\theta}}$ is the time derivative of the vector of temperatures at nodes, $\boldsymbol{\theta}$.

Finally, by combining equations (23) and (30), a system of differential algebraic equations (DAE), i.e. a thermal network, may be finally deduced from the heat equation:

$$
\mathbf{C} \dot{\boldsymbol{\theta}}=-\mathbf{A}^{T} \mathbf{G A \theta}+\mathbf{A}^{T} \mathbf{G b}+\mathbf{f}
$$




\section{Conclusions}

Thermal networks may be deduced from the heat equation by using the finite element method. This implies the statement of a theoretical framework to the use of thermal networks without considering the thermal-electrical analogy. Basically, the procedure consists on transforming the resolution of the heat equation into the resolution of a finite system of differential and algebraic equations, i.e. in the resolution of a thermal network. This enables the representation of the heat transfer problem in matrix form and facilitates the use of linear algebra for studying the dynamics of thermal systems, i.e. for solving parameter identification, simulation, and prediction or control problems. The method is to be used in practical applications where only a finite number of function values (i.e. discrete functions in space) are known.

\section{Acknowledgement}

Funding for this project was provided by a grant from la Région Rhône-Alpes, by the Spanish Ministry of Economy and Competitiveness within the program Juan de la Cierva-Formación and under project TIN2015-64776-C3-1-R.

\section{References}

[1] J. B. J. Fourier, Théorie analytique de la chaleur, 1822.

[2] T. N. Narasimhan, "Fourier's heat conduction equation: History, influence, and connections," Reviews of Geophysics, vol. 37, no. 1, pp. 151-172, 1999.

[3] H. Carslaw and J. Jaeger, Conduction of Heat in Solids, Clarendo Press, 1986.

[4] F. P. Incropera, Fundamentals of Heat and Mass Transfer, John Wiley \& Sons, 2006.

[5] A. Rabl, "Parameter Estimation in Buildings: Methods for Dynamic Analysis of Measured Energy Use," Journal of Solar Energy Engineering, vol. 110, no. 1, pp. 52-66, 1988.

[6] M. Milman and W. Petrick, "A note on the solution to a common thermal network problem encountered in heat-transfer analysis of spacecraft," Applied Mathematical Modelling, vol. 24, no. 12, pp. 861-879, 2000.

[7] Y. Chen, A. K. Athienitis and K. E. Galal, "Frequency domain and finite difference modeling of ventilated concrete slabs and comparison with field measurements: Part 1, modeling methodology," International Journal of Heat and Mass Transfer, vol. 66, pp. 948-956, 2013.

[8] G. Strang, Introduction to Applied Mathematics, Wellesley-Cambridge Press, 1986.

[9] G. Strang, Computational Science and Engineering, Wellesley-Cambridge Press, 2007.

[10] C. Ghiaus, "Causality issue in the heat balance method for calculating the design heating and cooling load," Energy, vol. 50, pp. 292-301, 2013. 
[11] I. Naveros and C. Ghiaus, "Order selection of thermal models by frequency analysis of measurements for building energy efficiency estimation," Applied Energy, vol. 139, pp. 230-244, 2015.

[12] I. Naveros, C. Ghiaus, D. Ruíz and S. Castaño, "Physical parameters identification of walls using ARX models obtained by deduction," Energy and Buildings, vol. 108, pp. 317-329, 2015. 